\title{
Surface Tension of Ionic Liquids Analogues Using the QSPR Correlation
}

\author{
F. S. Mjalli, K. Shahbaz, M. A. Hashim, and I. M. AlNashef
}

\begin{abstract}
Deep eutectic solvents (DESs) are mixture complexes being introduced in many applications due to their favorable physicochemical characteristics. However, due to the lack of experimental data, prediction of their physical properties is challenging. One of the important physical properties that provides considerable insight of the molecular influence on intensity of interactions in the mixture is the surface tension. In this work, the QSPR prediction method was employed to predict the DESs surface tension. The parachors of selected DESs based on ammonium and phosphunium salts were determined experimentally and also calculated from the molecular structure of their constituting components using available parachor contribution data for neutral compounds. The results showed that the calculated and experimental parachors of DESs were notably comparable and the parachor contribution data developed for neutral compounds can be successfully utilized for DESs. The calculated parachors were employed to predict the surface tension using their experimental densities values.
\end{abstract}

Index Terms-Surface tension, deep eutectic solvents, prediction.

\section{INTRODUCTION}

The growing number of publications in Deep Eutectic Solvents (DESs) literature reveals that they are gaining much attention by the scientific community. This is due to their potential as environment friendly solvents and advantages over conventional solvents and ionic liquids such as non-toxicity, non-reactivity with water and above all being biodegradable [1]. Moreover, they are synthesized easily in high purity at low cost in contrast to Ionic liquids which are very expensive and difficult to handle [2]. One of the fascinating features of using DESs is their potential as tunable solvents that can be customized to a particular type of chemistry. Surface tension is one of the essential DESs physical properties. Its evaluation is necessary to resolve various industrial related problems and develop new separation technologies. The awareness of this property provides considerable information of the molecular influence on the intensity of interactions in the mixture. Furthermore, the surface tension plays an important role in mass transfer processes like distillation, absorption, separation and extraction [3]. The experimental values for DESs surface

Manuscript received January 5, 2013; received March 9, 2013.

F. S. Mjalli is with Petroleum and Chemical Engineering Department, Sultan Qaboos University, Oman (e-mail: farouqsm@ yahoo.com).

K. Shahbaz is with the School of Engineering, Taylor's University, 47500 Selangor, Malaysia (e-mail: shahbaz.kaveh@ gmail.com).

M. A. Hashim is with University of Malaya, Chemical Engineering Department, Kuala Lumpur, Malaysia (e-mail: alihashim@um.edu.my).

I. M. AlNashef is with King Saud University, Chemical Engineering Department, Riyadh, Saudi Arabia (e-mail: alnashef@ksu.edu.sa). tension are either rare or even absent. It is also impractical to experimentally evaluate surface tension in many cases. Thus, prediction methods for surface tension of DESs are required. For this purpose the theoretical or empirical methods must be examined to find an acceptable and accurate method for prediction of surface tension. Due to the large potential number of DESs in various salts and hydrogen bond donors (HBD) combinations; it is not practical to find a unique correlation for predicting DESs surface tension. A desirable method for prediction of DESs surface tension and even DESs physical properties could be through their structures e.g. using quantitative structure property relationship (QSPR) [4].

Several methods have been suggested for prediction of the surface tension. Amongst them the empirical formula recommended by Macleod is the uncomplicated method [5]. This method expresses the temperature-independent relationship between surface tension and density of liquid, eq. 1 :

$$
\sigma^{1 / 4}=K . \rho
$$

where $\sigma$ is surface tension, $\rho$ is density and $K$ is a constant which is temperature independent. Sugden [6] modified the Macleod equation by multiplying each side of equation with molecular weight $\left(M_{w}\right)$ to give a constant which is called parachor $(P)$, Eq. 3:

$$
\begin{aligned}
& M_{w} \cdot \sigma^{1 / 4}=M_{w} \cdot K \cdot \rho \\
& P=M_{w} \cdot K=\frac{M_{w} \cdot \sigma^{1 / 4}}{\rho}
\end{aligned}
$$

Indeed, the parachor is a link between the surface tension, density and structure of a compound [7]. The parachor of a compound is the sum of its parachor contribution [6]. It is clear from Eq. 3 that through the parachors of a compound, the compound surface tension is predictable from knowledge of density and vice versa.

Mumford et al. [8] and Quayle [9] improved Sugden' original parachor contribution values for neutral compounds. In addition, Knotts et al. [10] developed a QSPR correlation for the parachors using the huge amount of physical data available for neutral organic compounds in the DIPPR database [11]. Knotts et al. [10] reported two individual parachor contribution data sets; the first data set was achieved using a training set containing experimental surface tension values with an uncertainty less than 5\% and the second was obtained using surface tension data with uncertainties of less than $1 \%$.

Although the parachor correlation proposed by Knotts et al. [10] has been considered for uncharged compound and no investigations were performed to develop QSPR correlation 
for ions, recently Deetlefs et al. [12] attempted to employ the Knotts's parachor correlation for prediction of ionic liquids surface tension. They calculated parachors of ionic liquids using the neutral parachor contribution values and revealed that the differences between the corresponding experimental and calculated parachor values were sufficiently small. Then using the calculated parachor values they predicted surface tension of ionic liquids from densities and vice versa. Consequently, they suggested that the QSPR correlation could be used for salts with electrostatic interactions in the salts. Afterwards, Gardas et al. [13] indicated that the QSPR correlation for parachors suggested by Knotts et al. [10] can be successfully extended for ionic liquids. They utilized the QSPR correlation along with a density prediction method proposed by themselves for estimation of ionic liquids surface tension in wide range of temperature (268.15-393.15 K) [14]. For validating the QSPR correlation for ionic liquids, they compared the predicted surface tension with the experimental values collected from literature and a database for ionic liquids surface tension.

To predict the surface tension of deep eutectic solvents by using Eq. (3), density data are required. Since density data is unavailable in many cases, lately, we have reported an empirical group contribution method to predict densities of DESs [1]. In that method the critical properties of salt and HBD were estimated separately using the modified Lydersen-Joback-Reid method, while that of the mixture were calculated using Lee-Kesler equation. The Rackett equation modified by Spencer and Danner was employed to predict the DES density. Moreover, newly, we developed an artificial neural network model to predict densities of three classes of deep eutectic solvents based on - phosphonium and ammonium salts [15].

Since there are no studies dedicated for the prediction of
DESs surface tension, the QSPR correlation proposed by Knotts et al. [10] will here be investigated for its ability to predict surface tension of DESs. In this work, nine DESs at different salts to HBD molar compositions will be considered for calculating their parachors that consequently will be used to predict their surface tension.

\section{EXPERIMENTAL}

\section{A. Chemicals}

Methyltriphenylphosphonium bromide $\left(\mathrm{C}_{19} \mathrm{H}_{18} \mathrm{PBr}\right)$, Choline chloride $\left(\mathrm{C}_{5} \mathrm{H}_{14} \mathrm{ClNO}\right)$ and $\mathrm{N}, \mathrm{N}$-Diethylenethanol ammonium chloride $\left(\mathrm{C}_{6} \mathrm{H}_{16} \mathrm{ClNO}\right)$ as salts and glycerol $\left(\mathrm{C}_{3} \mathrm{H}_{8} \mathrm{O}_{3}\right)$, ethylene glycol $\left(\mathrm{C}_{2} \mathrm{H}_{6} \mathrm{O}_{2}\right)$, triethylene glycol $\left(\mathrm{C}_{6} \mathrm{H}_{14} \mathrm{O}_{4}\right)$, 2,2,2-trifluoroacetamide $\left(\mathrm{C}_{2} \mathrm{H}_{2} \mathrm{~F}_{3} \mathrm{NO}\right)$ and 1 , 4-Butanediol $\left(\mathrm{C}_{4} \mathrm{H}_{10} \mathrm{O}_{2}\right)$ as hydrogen bond donors were purchased from Merck Chemicals with high purity ( $\geq 98 \%$ ) and utilized for the synthesis of DESs without further purification

\section{B. DESs Synthesis}

In this study, three different salts namely; Choline chloride, N,N-Diethylenethanol ammonium chloride and methyltriphenylphosphonium bromide and five different HBD namely; glycerol, ethylene glycol, triethylene glycol, 2,2,2-trifluoroacetamide and 1, 4-Butanediol were selected to synthesize the nine DESs with different compositions. The deep eutectic solvents were synthesized by mixing the salt with different HBD in different mole fraction of salt under an argon atmosphere and at $353.15 \mathrm{~K}$ until a homogenous and colorless liquid appeared. Table I presents the compositions of the different DESs synthesized in this study.

TABALE I: Compositions AND ABbREVIATION OF THE SyNTHESIZED DEEP EuteCTIC SOlVENTS.

\begin{tabular}{llccc}
\hline & HBD & $x_{\text {salt }}$ & $x_{H B D}$ & Abbreviation \\
\hline \multirow{3}{*}{ Choline chloride } & Glycerol & 0.33 & 0.67 & DES1 \\
& Ethylene glycol & 0.33 & 0.67 & DES2 \\
& 1, 4-Butanediol & 0.25 & 0.75 & DES3 \\
\hline \multirow{3}{*}{ Methyltriphenylphosphonium bromide } & Glycerol & 0.25 & 0.75 & DES4 \\
& Ethylene glycol & 0.20 & 0.80 & DES5 \\
& Triethylene glycol & 0.16 & 0.84 & DES6 \\
\hline \multirow{3}{*}{ N,N-Diethylenethanol ammonium chloride } & Glycerol & 0.20 & 0.80 & DES7 \\
& Ethylene glycol & 0.25 & 0.75 & DES8 \\
& 2,2,2-Trifluoroacetamide & 0.33 & 0.67 & DES9 \\
\hline
\end{tabular}

\section{DESs Characterization}

The freezing point of all DESs were measured using Mettler Toledo Differential Scanning Calorimeter) DSC 1 STAR $^{\mathrm{e}}$ System) which was calibrated against known standards (water and 2-propanol) to ensure the measurement's accuracy. Furthermore, the water content of all DESs was determined by Karl Fisher titration method and the measurement accuracy of the Karl Fisher coulometer was checked with Hydranal-water standard $1.00 \mathrm{mg} / \mathrm{g}$. This standard was supplied in glass ampoules from Sigma-Aldrich, Malaysia. Table II shows the freezing temperature and water content of studied DESs in this work.

Densities of the synthesized DESs in this study were determined using a DMA 4500 vibrating tube density/specific gravity meter (Anton Paar, Austria). To check the density meter adjustment, density of water (degassed bi-distillated) was measured at $298.15 \mathrm{~K}$ and compared with the corresponding value in the density tables [16]. The results showed a difference of $\pm 0.00003 \mathrm{~g} \mathrm{~cm}^{-3}$ which confirmed the accuracy of machine. Density measurements were performed at $298.15 \mathrm{~K}$ with three replicates for each reading, the uncertainty in density measurements was $\pm 0.00005 \mathrm{~g} \cdot \mathrm{cm}^{-3}$. 


\begin{tabular}{ccc}
\multicolumn{2}{c}{ TABAL II: FREEZING TEMPERATURES AND WATER CONTENT FOR } \\
SYNTHESIZED DEEP EUTECTIC SOLVENTS.
\end{tabular}

The surface tension measurements of all DES were carried out using a Krüss K100 tensiometer by the Du Noüy ring method [17]. The tensiometer was equipped with LabDesk 3.2 software for data collation and automatic control. The platinum-iridium ring was cleaned by flaming and the glassware was rinsed consecutively with acetone and distillated water before beginning each measurement. The calibration of equipment was determined by measuring the surface tension of pure water before using. The Surface tensions of all studied DESs were measured at $298.15 \mathrm{~K}$ and for each measurement three replicates were conducted and the uncertainties of the surface tension and temperature values were within the range of $\pm 0.2 \mathrm{mN} \mathrm{m}^{-1}$ and $\pm 0.1 \mathrm{~K}$, respectively.

\section{RESULTS AND DISCUSSION}

The nine different DESs based on ammonium and phosphonium salts were selected and synthesized to test the QSPR method for predicting the surface tension. The mole fractions of salt and HBD used to synthesize each DES in this work were selected based on the lowest freezing temperature attained for DES. As can be seen from Table II, all synthesized DESs (DES1 to DES9) had low freezing temperatures as compared to their constituting components and were liquids at ambient temperature. This is a main characteristic of deep eutectic solvents. Furthermore, water content, which has a significant effect on the physical properties of deep eutectic solvents, of all DESs (DES1 to DES9) was less than $0.1 \mathrm{wt} \%$ (Table II) which is comparable to that reported in the literature.

Table III lists the molecular weights and measured densities and surface tensions of all studied DESs in this work at $298.15 \pm 0.1 \mathrm{~K}$. Since impurities like water affect physical property values, all measurements for each DES were carried out with the same sample to ensure reproducibility. Due to lack of studies of DESs physical properties, it was not possible to compare our experimental results of the surface tension of DESs. However, Harris [18] reported data for the surface tension of some choline chloride-based DESs as function of temperature. The surface tension value reported for choline chloride:glycerol DES (DES1) at 0.33 mole fraction of choline chloride and at $298.15 \mathrm{~K}_{\text {was }} 55.4 \mathrm{mN} \mathrm{m}^{-1}$ which is close enough to the value obtained in this work, i.e. $57.24 \mathrm{mN} \mathrm{m}^{-1}$. In addition, our measured surface tension value of $47.17 \mathrm{mN} \mathrm{m}^{-1}$ for DES3 (Choline chloride: 1,

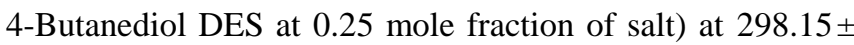
$0.1 \mathrm{~K}$ is slightly lower than that reported by Harris (2008) of $47.4 \mathrm{mN} \mathrm{m}^{-1}$. These differences in surface tension values might be due to the difference in water content in samples of Harris work and this work. The values of measured densities of DES2, DES5, DES8 and DES9 are almost equal to that reported in our previous work [19].

To calculate the parachor of each DES, the parachors of constituting individual components (salt and HBD) of each DES were calculated using the published parachor contribution data [10]. In this study, only the accurate parachor data set (with uncertainties of less than 1\%) proposed by Knotts et al. [10] were employed. After calculation the parachors of salt and HBD of each DES, the Eq. 4 was employed for calculation of DES parachor.

$$
P=\Sigma x_{i} P_{i}
$$

where $x_{i}$ refers to the mole fraction of the pure constituent and $P_{i}$ refers to parachor of pure constituent.

TABLE III: MEASURED DENSITIES AND SURFACE TENSIONS OF THE STUDIED DESS AT $298.15 \pm 0.1 \mathrm{~K}$.

\begin{tabular}{cccc}
\hline & & $\rho_{\exp }\left(\mathrm{g} \mathrm{cm}^{-3}\right)$ & $\sigma_{\exp }\left(\mathrm{mN} \mathrm{m}^{-1}\right)$ \\
\cline { 3 - 4 } & & $T / \mathrm{K}=298.15$ & $T / \mathrm{K}=298.15$ \\
\hline 2 & 107.94 & 1.1920 & 57.24 \\
3 & 87.92 & 1.1168 & 48.91 \\
4 & 102.49 & 1.0610 & 47.17 \\
5 & 158.38 & 1.2976 & 58.94 \\
6 & 121.10 & 1.2325 & 51.29 \\
7 & 184.68 & 1.1858 & 49.85 \\
8 & 104.41 & 1.2201 & 59.35 \\
9 & 84.96 & 1.1014 & 47.51 \\
\hline
\end{tabular}

Table IV shows the calculated parachors for all DESs (DES1 to DES9) using Eq.4 and experimental parachors. The experimental parachor of each DES was determined using Eq.3 through the measured density and surface tension of DES. In addition the percentage of errors ( $\%$ E) obtained using Eq. 5 are given in Table IV.

$$
\% E=\frac{\text { Value }(\text { Experimental }- \text { calculated })}{\text { Value }(\text { Experimental })} \times 100
$$

As indicated by Table IV, the percentage errors obtained for calculated and experimental parachors of all DES were very small. Moreover, a comparative plot (Fig. 1) of calculated parachors against experimental parachors for studied DESs revealed that experimental and calculated parachores value were extremely correlated $\left(R^{2}=0.9987\right)$ and highly similar. Accordingly, there is a good agreement for employing the parachor contribution data for prediction of surface tension of deep eutectic solvents, despite the fact that 
parachor contribution data proposed by Knotts et al. [10] were considered based on covalent bonds and did not take into account coordinate bonds.

TABLE IV: CALCULATED PARACHORS AND PREDICTED SURFACE TENSIONS OF THE STUDIED DESS.

\begin{tabular}{ccccccc}
\hline \multirow{7}{*}{ DES } & $P_{\text {exp }}$ & $P_{\text {cal }}$ & $\% E$ & $\begin{array}{c}\sigma_{\text {exp }} \\
T / \mathrm{K}\end{array}$ & $\begin{array}{c}\sigma_{p} \\
T / \mathrm{K}\end{array}$ & $\% E$ \\
\hline \multirow{2}{*}{1} & 249.0 & 246.7 & \multirow{2}{*}{0.94} & 57.2 & 55.0 & 3.74 \\
& 7 & 1 & & 4 & 9 & \\
2 & 208.1 & 205.0 & & 48.9 & 46.0 & 5.91 \\
& 9 & 4 & 1.51 & 1 & 1 & \\
& 253.1 & 248.6 & & 47.1 & 43.9 & 6.89 \\
3 & 6 & 8 & 1.77 & 7 & 1 & \\
& 338.1 & 335.5 & & 58.9 & 57.1 & 3.09 \\
4 & 9 & 5 & 0.78 & 4 & 1 & \\
& 262.9 & 258.9 & & 51.2 & 48.2 & 5.90 \\
5 & 4 & 8 & 1.50 & 9 & 6 & \\
& 413.8 & 406.7 & & 49.8 & 46.5 & 6.70 \\
6 & 3 & 1 & 1.71 & 5 & 0 & \\
& 237.5 & 237.0 & & 59.3 & 58.9 & 0.73 \\
7 & 0 & 8 & 0.18 & 5 & 1 & \\
& 202.5 & 198.8 & & 47.5 & 44.1 & 7.14 \\
8 & 2 & 0 & 1.83 & 1 & 1 & 14.8 \\
& 246.5 & 236.8 & & 40.2 & 34.2 & 14.93 \\
9 & 2 & 1 & 3.93 & 7 & 9 & 4 \\
\hline
\end{tabular}

Table IV also illustrates the experimental DESs surface tensions and their corresponding predicted values. The calculated parachor for each DES was employed in Eq. 3 for predicting the DES surface tension using its experimental density data. The value of the average percentage error for all studied DESs was 6.4\%. This shows that using the QSPR correlation method for prediction of DESs surface tensions is appropriate. In addition, it is also shown in Fig. 2 that the experimental surface tension values of DESs exhibit linear relationship with predicted DESs surface tensions $\left(\mathrm{R}^{2}=\right.$ 0.9952). However, the percentage errors in surface tensions are bigger than that in the corresponding parachors. As results indicated in Table $\mathrm{V}$, the percentage error in prediction of DESs surface tension for glycerol-based DESs is lower than that of other DESs. This could be attributed to the fact that the values of the surface tensions of glycerol-based DESs were higher in values than that for other classes of DESs and it can be concluded that the QSPR correlation method has high accuracy for prediction of DESs surface tensions with high values.

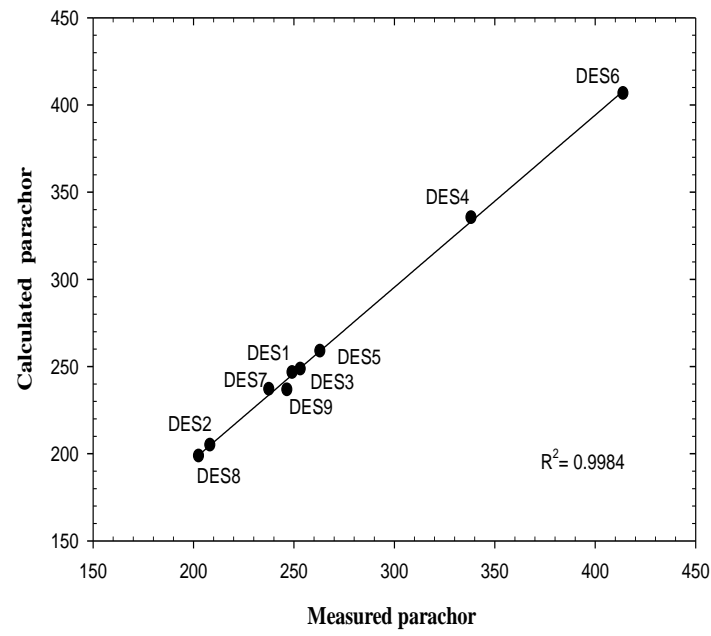

Fig. 1. A plot of the experimental versus calculated DESs parachors.

By means of the same methodology for predicting DESs surface tensions, the densities of the studied DESs were also predicted using Eq. (3) and employing the calculated parachors and experimental DESs surface tension values. Table $\mathrm{V}$ shows the measured and predicted DESs densities and percentage errors in densities of the studied DESs.

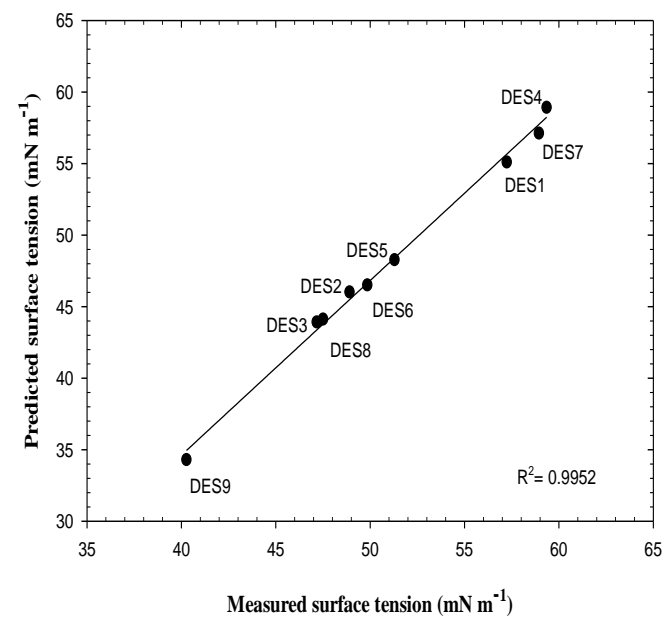

Fig. 2. A plot of measured versus predicted (using QSPR correlation method) surface tension of the studied DESs.

A plot of experimental DESs densities against the corresponding predicted values (Fig. 3) reveals that the values of the predicted densities were in good agreement with the experimental values. Again, this proves that using calculated parachors to predict the DESs densities is an appropriate method even though parachors were calculated from neutral parachor contribution data.

TABLE V: PREDICTED AND MEASURED DENSITIES OF THE STUDIED DESS.

\begin{tabular}{cccc}
\hline DES & \multicolumn{1}{l}{$\rho_{\text {exp }}$} & \multicolumn{1}{l}{$\rho_{p}$} & $\% E^{a}$ \\
\hline 1 & 1.1920 & 1.2034 & 0.96 \\
2 & 1.1168 & 1.1339 & 1.54 \\
3 & 1.0610 & 1.0801 & 1.80 \\
4 & 1.2976 & 1.3078 & 0.79 \\
5 & 1.2325 & 1.2514 & 1.53 \\
6 & 1.1858 & 1.2065 & 1.75 \\
7 & 1.2201 & 1.2223 & 0.18 \\
8 & 1.1014 & 1.1220 & 1.87 \\
9 & 1.2934 & 1.3464 & 4.10 \\
\hline
\end{tabular}

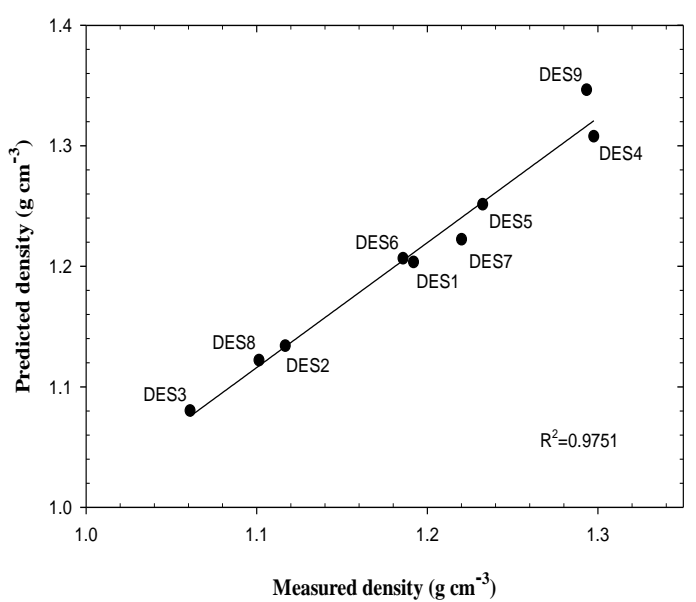

Fig. 3. Comparison between the measured and predicted (using QSPR correlation method) DESs densities. 


\section{CONCLUSIONS}

This study introduced a simple method for prediction of surface tension of deep eutectic solvents. In this method the parachor plays an important role as a reliable predictive link between the surface tension and density of DESs and their molecular structures. The nine different DESs based on ammonium and phosphunium salts were selected to calculate their parachors using neutral parachor contribution data. The results revealed that the difference between calculated and experimental parachors of DESs was adequately small and this showed clearly that the parachor contribution data developed for neutral compounds can be successfully utilized for deep eutectic solvents. Furthermore, using calculated parachors and experimental DESs densities values the surface tensions of DESs were predicted with an average percentage error of $6.4 \%$. The results also revealed that using parachor, the densities of DESs can be predicted from their surface tensions.

\section{REFERENCES}

[1] K. Shahbaz, F. S. Mjalli, M. A. Hashim, and I. M. Alnashef, "Using Deep Eutectic Solvents Based on Methyl Triphenyl Phosphunium Bromide for the Removal of Glycerol from Palm-Oil-Based Biodiesel," Energy and Fuels, vol. 25, pp. 2671-2678, 2011.

[2] Y. Hou, Y. Gu, S. Zhang, F. Yang, H. Ding, and Y. Shan, "Novel binary eutectic mixtures based on imidazole," Journal of Molecular Liquids, vol. 143, pp. 154-159, 2008.

[3] E. Jimenez, M. Cabanas, L. Segade, S. Garca-Garabal, and H. Casas, "Excess volume, changes of refractive index and surface tension of binary 1,2-ethanediol + 1-propanol or 1-butanol mixtures at several temperature," Fluid Phase Equilibria, vol. 180, pp. 151-164, 2001.

[4] A. R. Katritzky, V. S. Lobanov, and M. Karelson, "QSPR: the correlation and quantitative prediction of chemical and physical properties from structure," Chemical Society Reviews, vol. 24, pp. 279-287, 1995.

[5] D. B. Macleod, "On a relation between surface tension and density," Transactions of the Faraday Society, vol. 19, pp. 38-41, 1923.

[6] S. Sugden, "VI.-The variation of surface tension with temperature and some related functions," Journal of the Chemical Society, Transactions, vol. 125, pp. 32-41, 1924.
[7] J. R. Parthington, "An Advanced Treatise on Physical Chemistry," London, Longmans, Green and Co, 1951.

[8] S. A. Mumford and J. W. C. Phillips, "CCLXXIV.-The evaluation and interpretation of parachors," Journal of the Chemical Society (Resumed), pp. 2112-2133, 1929.

[9] O. R. Quayle, "The Parachors of Organic Compounds. An Interpretation and Catalogue," Chemical Reviews, vol. 53, pp. 439-589, 1953.

[10] T. A. Knotts, W. V. Wilding, J. L. Oscarson, and R. L. Rowley, "Use of the DIPPR Database for Development of QSPR Correlations: Surface Tension," Journal of Chemical \& Engineering Data, vol. 46, pp. 1007-1012, 2001.

[11] CrossFire 2000, Version 5.0, Database BS0203AB, Beilstein.

[12] M. Deetlefs, K. R. Seddon, and M. Shara, "Predicting physical properties of ionic liquids," Physical Chemistry Chemical Physics, vol. 8, pp. 642-649, 2006.

[13] R. L. Gardas and J. O. A. P. Coutinho, "Applying a QSPR correlation to the prediction of surface tensions of ionic liquids," Fluid Phase Equilibria, vol. 265, pp. 57-65,2008.

[14] R. L. Gardas and J. O. A. P. Coutinho, "Extension of the Ye and Shreeve group contribution method for density estimation of ionic liquids in a wide range of temperatures and pressures," Fluid Phase Equilibria, vol. 263, pp. 26-32, 2008.

[15] K. Shahbaz, S. Baroutian, F. S. Mjalli, M. A. Hashim, and I. M. Alnashef, "Densities of ammonium and phosphonium based deep eutectic solvents: prediction using artificial intelligent and group contribution techniques," Thermochimica Acta, vol. 527, pp. 59-66, 2011.

[16] H. Bettin and F. Spieweck , "Die Dichte des Wassers als Funktion der Temperatur nach Einführung der Internationalen Temperaturskala," PTB-Mitt, vol. 1, pp. 195-196, 1990.

[17] P. L. Du Noüy, "An Interfacial Tensiometer for Universal Use," The Journal of General Physiology, vol. 7, pp. 625-631, 1925.

[18] R. C. Harris, "Physical Properties of Alcohol Based Deep Eutectic Solvents," Department of Chemistry Leicester, University of Leicester, 2008.

[19] K. Shahbaz, F. S. Mjalli, M. A. Hashim, and I. M. Alnashef, "Prediction of deep eutectic solvents densities at different temperatures," Thermochimica Acta, vol. 515, pp. 67-72, 2011.

Farouq S. Mjalli is an associate professor at the Department of Petroleum and Chemical Engineering, Faculty of Engineering, Sultan Qaboos University, Oman. 